\title{
Phase Behavior of Undecane-Dodecane Mixtures Confined in SBA-15
}

\author{
Xiao Yan, ${ }^{1}$ Hai-Rong Pei, ${ }^{1}$ Tong-Bo Wang, ${ }^{1}$ Wen-Bo Liu, ${ }^{2}$ and Xiao-Zheng Lan' \\ ${ }^{1}$ College of Chemistry and Materials Science, Shandong Agricultural University, Shandong, Taian 271018, China \\ ${ }^{2}$ Binzhou Medical College, Shandong, Yantai 264003, China \\ Correspondence should be addressed to Xiao-Zheng Lan; lanxzh@sdau.edu.cn
}

Received 23 June 2012; Revised 20 July 2012; Accepted 30 July 2012

Academic Editor: Nianjun Yang

Copyright (C) 2013 Xiao Yan et al. This is an open access article distributed under the Creative Commons Attribution License, which permits unrestricted use, distribution, and reproduction in any medium, provided the original work is properly cited.

\begin{abstract}
Phase behavior of undecane-dodecane mixtures $\left(n-\mathrm{C}_{11} \mathrm{H}_{24}-\mathrm{C}_{12} \mathrm{H}_{26}, \mathrm{C}_{11}-\mathrm{C}_{12}\right)$ in bulk and confined in SBA-15 (pore diameters 3.8 , 7.8 , and $17.2 \mathrm{~nm}$ ) has been studied by differential scanning calorimetry. Phase diagram of the confined $\mathrm{C}_{11}-\mathrm{C}_{12}$ system shows dependence on pore size, indicating simple behavior compared with the bulk. In the three systems, melting points of the confined $\mathrm{C}_{11}, \mathrm{C}_{12}$ and the mixtures $\left(x_{\mathrm{C} 12}=0.1-0.9\right)$ present a linear relation with the pore diameter from 3.8 to $17.2 \mathrm{~nm}$. Melting behavior of the confined $\mathrm{C}_{11}-\mathrm{C}_{12}$ mixtures is closely as the confined $\mathrm{C}_{11}, \mathrm{C}_{12}$. Melting temperatures of the three systems have been fitted as a function of mole fraction $x_{\mathrm{C} 12}$.
\end{abstract}

\section{Introduction}

Study on melting and freezing of fluids confined in nanopores materials is important from the theoretical as well as practical point of view [1-5]. The research in this field aims at understanding the influence of size effect and interface interactions on materials $[6,7]$. It was found that melting temperatures of some liquids, such as water confined in CPG [6], follow the Gibbs-Thomson (G-T) equation. However, when the pore is very small or pore wall changed, such as activated carbon, carbon nanotubes, and mica, melting points of confined liquids deviated from the G-T equation or even lifted [6-10]. The new phenomena were thought to be related to interface interactions $[6,11]$.

Meanwhile, few investigations have been reported on phase behavior of binary mixtures confined in nanopores. It was found that $\mathrm{CaCl}_{2}-\mathrm{H}_{2} \mathrm{O}$ mixtures confined in silica gel (pore diameter, $15 \mathrm{~nm}$ ) belong to the same eutectic system with that for the bulk, whereas the phase diagram was shifted down by $10-30 \mathrm{~K}$ [12]. A similar phenomenon was observed in $\mathrm{C}_{6} \mathrm{H}_{5} \mathrm{Br}_{-} \mathrm{CCl}_{4}$ system confined in CPGs with pore diameter $7.5 \mathrm{~nm}$ [13]. The size effect shows great influence on the phase behavior of $\mathrm{C}_{6} \mathrm{H}_{5} \mathrm{Cl}-\mathrm{C}_{6} \mathrm{H}_{5} \mathrm{Br}$ and $n-\mathrm{C}_{12} \mathrm{H}_{24}-\mathrm{C}_{14} \mathrm{H}_{30}$ mixtures adsorbed in mesoporous silicates [14-16]. At present, the influence of size effect and interface interactions on phase behavior of binary fluids is still not fully understood.

Normal alkanes and their mixtures are extensively investigated in petroleum industry. In theoretical aspect, they can offer a good model in study on complex crystallization behavior of polymer materials, surfactants and lipids, and so forth [17]. Among this family, undecane $\left(\mathrm{C}_{11}\right)$ and dodecane $\left(\mathrm{C}_{12}\right)$ have medium size carbon chains and same crystal structures [18]. Their crystals have a triclinic unit cell belonging to space group $P 1^{-}(Z=1)$ with dimensions $(a \approx 4.3$, $b \approx 4.8, c \approx 20) \AA\left(C_{11}\right)[19-21],(a=4.3, b=4.8, c=$ 17.3) $\AA\left(C_{12}\right)$ [18], respectively. $C_{11}-C_{12}$ bulk system displays a complicated phase behavior [22]. $\mathrm{C}_{11}-\mathrm{C}_{12}$ mixtures are only partial miscible in solid states.

SBA-15 has ordered cylindrical channels with twodimensional hexagonal arrangement. It is a new type of silicate mesoporous materials with potential applications in many fields. The tunable pores with narrow size distributions are the ideal adsorbent in study of confinement effect on fluids [23].

In this paper, we propose an investigation of phase behavior the odd-even $\mathrm{C}_{11}-\mathrm{C}_{12}$ alkane mixtures confined in SBA-15 (pore diameters 3.8, 7.8, and $17.2 \mathrm{~nm}$ ) by using DSC. Solid-liquid phase diagram of $\mathrm{C}_{11}-\mathrm{C}_{12} / \mathrm{SBA}-15$ showed 
dependence on the pore size. Moreover, the alkane mixture inside pores of SBA-15 behaves seemingly as the confined $\mathrm{C}_{11}, \mathrm{C}_{12}$.

\section{Experimental}

2.1. Materials. Undecane (mass fraction purity $\geq 0.98$ ), dodecane (mass fraction purity $\geq 0.98$ ), and tetraethyl orthosilicate (TEOS, mass fraction purity 0.999) were purchased from Aladdin Reagents Co., Shanghai, China. 1, 3, 5-Triisopropylbenzene (TIPB, mass fraction purity 0.97 ) was bought from Xiya Reagents Co., Chengdu, China. Triblock copolymer Pluronic P123 was obtained from Sigma. All chemicals were used as received without further purification.

2.2. Synthesis and Characterization of SBA-15. SBA-15 was synthesized according to literature methods, in which triblock copolymer Pluronic P123 was used as a template and TIPB as a micelle expander for large pore SBA-15 $(17.2 \mathrm{~nm})$ [24-26]. After degassed at $433 \mathrm{~K}$ for $6 \mathrm{~h}$, nitrogen adsorption and desorption isotherms for SBA-15 were measured at $77 \mathrm{~K}$ using an Autosorb-1 system [27].

Transmission electron microscopy (TEM) images were recorded on a JEM-1400 (JEOL) operated at $120 \mathrm{kV}$. Before imaging, SBA-15 powder was dispersed in ethanol through sonication and then deposited on a carbon-coated copper grid.

2.3. Sample Preparation and DSC Measurements. SBA-15 powder with a mass of about $10 \mathrm{mg}$ was put in a glass tube and outgas at $423 \mathrm{~K}$ under a vacuum of $10^{-1}$ Torr for around two hours. Then a certain amount of $\mathrm{C}_{11}, \mathrm{C}_{12}$ or their mixtures was transferred into the tube at pure nitrogen atmosphere. The volume of alkanes was taken as $90-100 \%$ the pore volume of SBA-15. At last, the glass tube was sealed and equilibrated at room temperature for about 6 hours.

SBA-15 with the alkane adsorbed was transferred into DSC aluminum pan and sealed immediately. The sample was analyzed on a TA DSC Q10 under a high purity nitrogen atmosphere at a scanning rate of $5 \mathrm{~K} \cdot \mathrm{min}^{-1}$. The temperature scale was calibrated by high purity indium and water for DSC Q10. The mass of samples for DSC analysis was about 1 to $3 \mathrm{mg}$ and measurement at least repeated three times in the temperature range from (173-190) K to room temperature. In most cases the melting temperature was reproducible to within $0.5 \mathrm{~K}$.

\section{Results and Discussion}

3.1. TEM Characterization and Pore Size of SBA-15. Three kinds of ordered mesoporous SBA-15 were synthesized with pore diameters of $3.8,7.8$, and $17.2 \mathrm{~nm}$, respectively, determined by BJH method on basis of adsorption branches of isotherms [16]. Pore volume of the three SBA-15 (3.8, 7.8, and $17.2 \mathrm{~nm}$ ) is $0.3,1.0$ and $1.4 \mathrm{~cm}^{3} \mathrm{~g}^{-1}$, respectively; micropore volume is determined to be $\sim 0,0.05$ and $0.02 \mathrm{~cm}^{3} \mathrm{~g}^{-1}$ by $V-t$ methods, respectively. As seen in Figure 1, SBA-15 presents parallel channels on TEM images and cylindrical mesopores with two-dimensional hexagonal arrangements ( $a, b$, and c). The pores show a uniform size under TEM imaging. After adsorbed in the channel of SBA-15, the alkanes would form one-dimensional liquid when the pore is nearly filled. The ordered mesopores in SBA-15 would make the DSC signals sharp. For further study, the regular pore structure should be advantageous when the pore wall of SBA-15 is modified.

3.2. Phase Behavior of $C_{11}-C_{12}, C_{11}-C_{12} / S B A-15$ (3.8, 7.8, and $17.2 \mathrm{~nm}$ ). Phase behavior of $\mathrm{C}_{11}-\mathrm{C}_{12}$ bulk system was investigated using differential scanning calorimetry. DSC curves of the mixtures at mole fractions of dodecane, $x_{\mathrm{C} 12}=$ $0-1$, are shown in Figure 2. The signals in low-temperature range (dash lines) are enlarged in aid of observation. Solidliquid phase diagram of $\mathrm{C}_{11}-\mathrm{C}_{12}$ system was determined by "shape factor method" [28] and shown in Figure 3, very close to the diagram in [22]. Different phases in the figure are designated by citing the results from the literature [22]. The phase diagram has shown two invariants, a peritectic and a eutectic point. The bulk system shows a relatively complicated behavior because of the special rotator phase in normal alkanes.

In Figure 4, DSC curves show the melting behavior of $\mathrm{C}_{11}-\mathrm{C}_{12}$ system confined in SBA-15 $(3.8 \mathrm{~nm})$. Only one endothermic peak was observed for all the samples, including $\mathrm{C}_{11}$ which has solid-liquid (s-l) and solid-solid (s-s) phase transitions in the bulk. To make sure the phenomena, the samples had been cooled down about $50 \mathrm{~K}$ below the melting point of each peak; however, no more thermal anomaly was found. Experimental phase diagram was established for $\mathrm{C}_{11^{-}}$$\mathrm{C}_{12} / \mathrm{SBA}-15(3.8 \mathrm{~nm})$ system on basis of the onset point of each peak as shown in Figure 5. The s-l boundary is a straight line, where melting points are proportional to $x_{\mathrm{C} 12}$. This is a system of miscible both in solid and liquid state according to classification of phase diagrams of bulk system. The behavior is very different with the bulk system.

When the pore size of SBA-15 comes to $7.8 \mathrm{~nm}$, the alkane adsorbed also displays a single endothermic melting peak on DSC curve scanned from very low temperature at mole fractions of $x_{\mathrm{C} 12}$ as shown in Figure 6. By onset points of these peaks, the experimental phase diagram of $\mathrm{C}_{11}-\mathrm{C}_{12} / \mathrm{SBA}-15$ system was decided as in Figure 7 . The diagram comprises of a curved line and also means a system of completely miscible both in solid and liquid state according to classification of phase diagrams of bulk system too.

As the pore diameter reaches $17.2 \mathrm{~nm}$, the alkanes inside channel of SBA-15 show some changing in melting behavior as shown in Figure 8. All the samples display melting peaks in higher-temperature region. In the low temperature range, $\mathrm{C}_{11}$ and the mixtures at $x_{\mathrm{C} 12}=0.1-0.4$ present solid-solid phase transitions, indicated as dash line next to the complete curve. Experimental phase diagram of the confined mixtures was determined on connection of onset points of the s-l and s-s transition peaks and shown in Figure 9. Perhaps, the absence in s-s boundary over the range $x_{\mathrm{C} 12}=0.5-1$ might result from incomplete crystallization or supercooling of the alkane mixtures. 


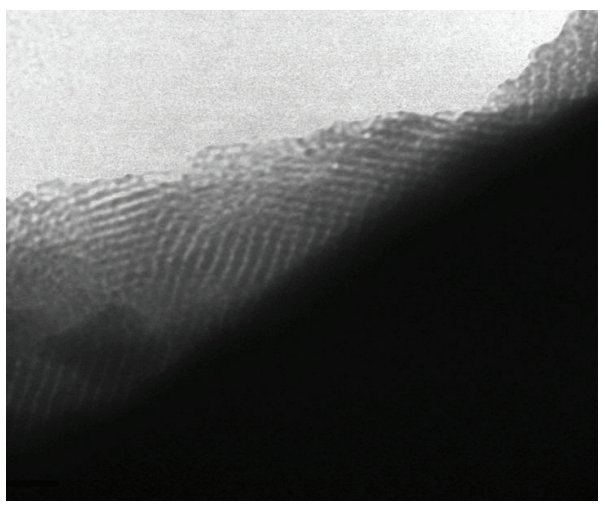

(a)

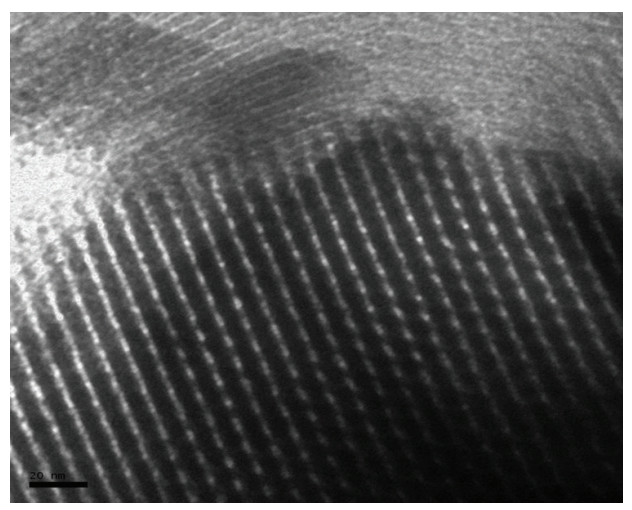

(b)

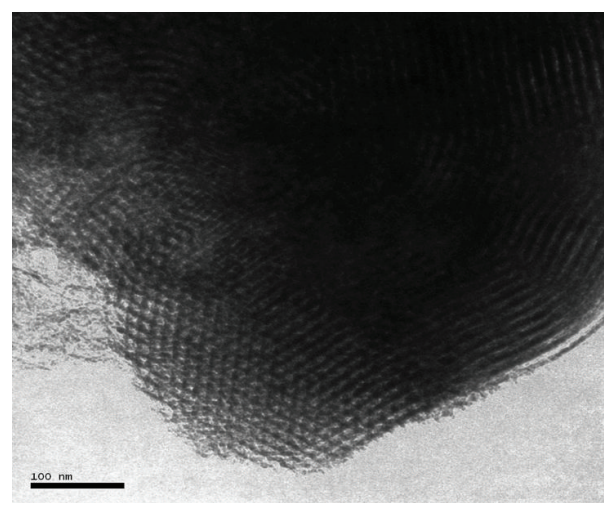

(c)

FIGURE 1: TEM images of SBA-15: (a) pore diameter $3.8 \mathrm{~nm}$; (b) $7.8 \mathrm{~nm}$; (c) $17.2 \mathrm{~nm}$. Scale bars are 50, 20, and $100 \mathrm{~nm}$, respectively.

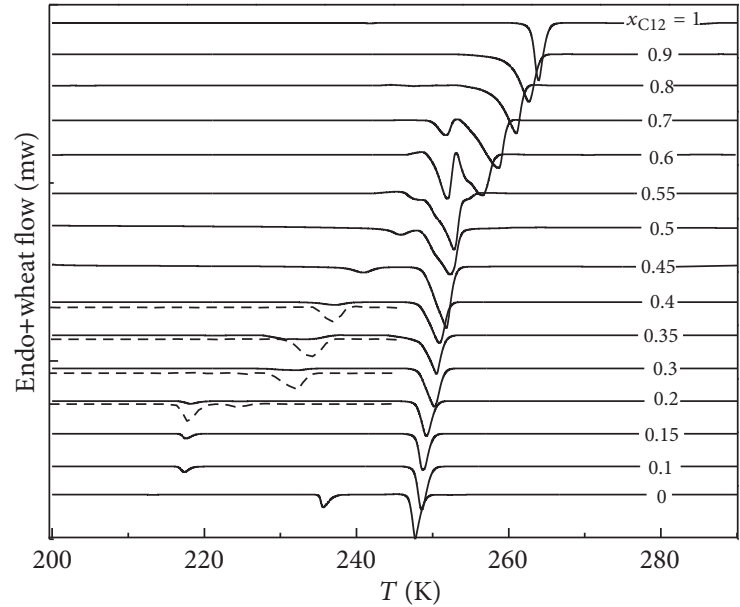

FIgURE 2: DSC curves of $\mathrm{C}_{11}-\mathrm{C}_{12}$ bulk system at different mole fractions of $\mathrm{C}_{12}\left(x_{\mathrm{C} 12}\right)$. The low-temperature region (dash line) is enlarged for easy observation.

3.3. Comparison of the Phase Behavior of $C_{11}-C_{12} / S B A-15$ (3.8, 7.8 , and $17.2 \mathrm{~nm}$ ). In preparation of samples, the volume of alkanes was added nearly the same as pore volume of SBA15. In analysis, no thermal anomalies from the bulk, pure alkanes, or the mixtures were observed on heat flow signals. It means that the alkanes should be adsorbed into pores of

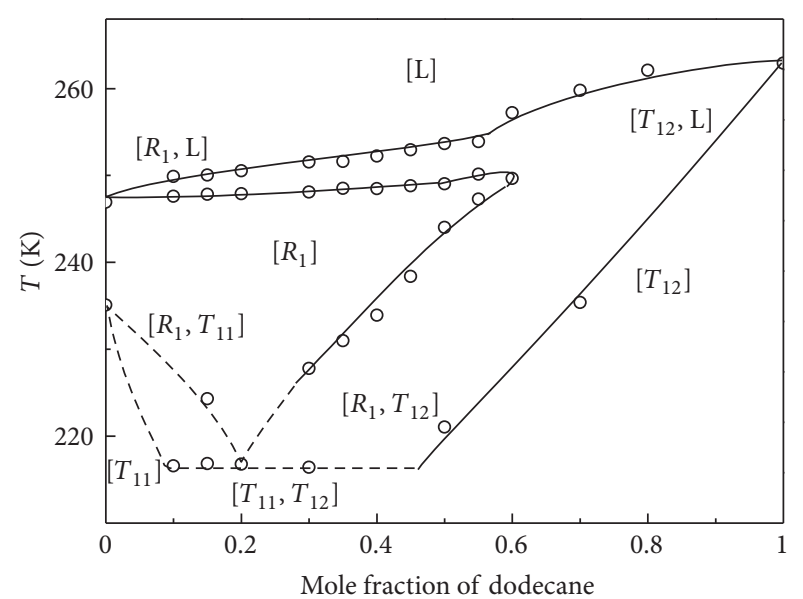

FIgURE 3: Experimental solid-liquid phase diagram of bulk $\mathrm{C}_{11}-\mathrm{C}_{12}$ system. $T$ represents triclinic crystal, $R$ rotator phase.

SBA-15 with no extra liquids left. In this case, interference of bulk alkane was avoided, and DSC signals reflected only properties of pore liquids. In addition, influence of equilibrium time and temperature on preparation of samples had been examined. There almost no difference in the melting points of samples equilibrated at room temperature to $323 \mathrm{~K}$ during a period about $1-6$ hours. It indicates that the alkanes entered pores of SBA-15 by wetting. In this condition, no 


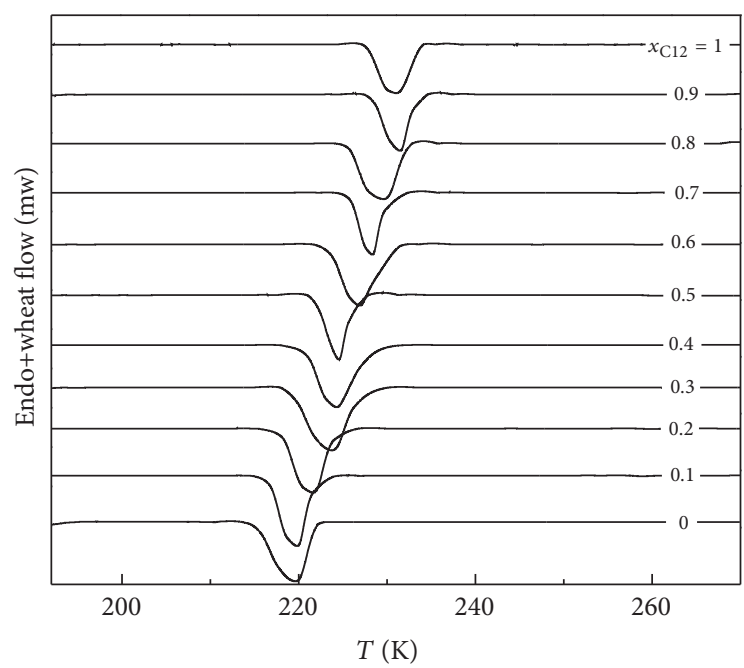

FIgURE 4: DSC curves of $\mathrm{C}_{11}-\mathrm{C}_{12}$ system confined in SBA-15 (pore diameter $3.8 \mathrm{~nm}$ ) as a function of mole fractions of $\mathrm{C}_{12}\left(x_{\mathrm{C} 12}\right)$.

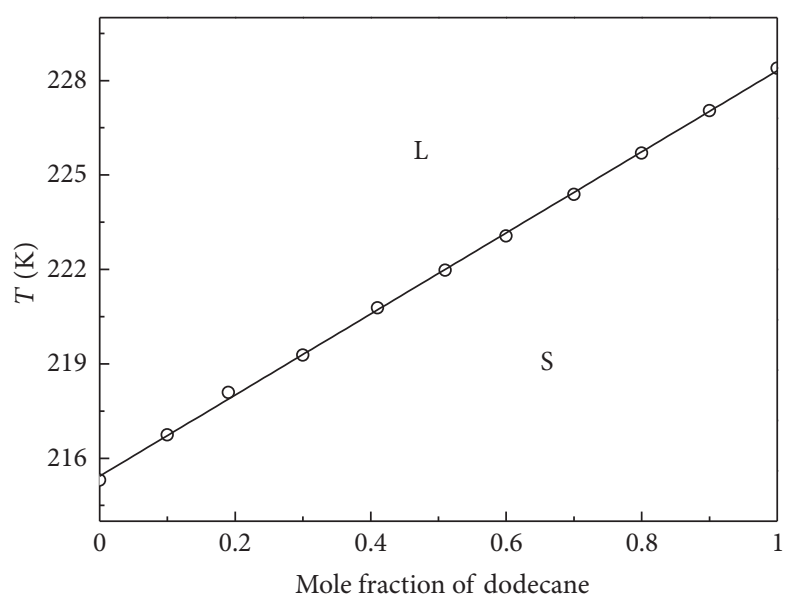

FIGURE 5: Experimental phase diagram of $\mathrm{C}_{11}-\mathrm{C}_{12}$ system confined in SBA-15 (3.8 nm).

discrimination in adsorption of $\mathrm{C}_{11}$ and $\mathrm{C}_{12}$ molecules exists inside pores of SBA-15 $[29,30]$, considering that the micropore volume takes a small portion in total pore volume. Accordingly, DSC analysis could provide correct information of $\mathrm{C}_{11}-\mathrm{C}_{12}$ binary mixtures in present experiments.

From Figures 5, 7, and 9, the development of phase diagram can be observed among the systems $\mathrm{C}_{11}-\mathrm{C}_{12} / \mathrm{SBA}-$ $15(3.8,7.8$, and $17.2 \mathrm{~nm})$. With the increasing of the size, phase diagram of the confined system grows from a straight line $(3.8 \mathrm{~nm}, \mathrm{~s}-1)$, a curve $(7.8 \mathrm{~nm}, \mathrm{~s}-1)$ to a loop line $(17.2 \mathrm{~nm}$, $\mathrm{s}$-l plus s-s). In the bulk scale, it would attain the complicated phase diagram showing all the specific characteristics of rotator phase in the system [22].

It is known that the cross-section of pure normal alkanes $(\sigma)$ is around $0.49 \mathrm{~nm}$ in the most stable configuration [20]. Therefore, SBA- 15 with pore diameter $(3.8,7.8$, and 17.2$) \mathrm{nm}$ has a channel of about $8 \sigma, 16 \sigma$, and $35 \sigma$ in radial direction of the pore. Perhaps, alkane molecules in pores of 3.8 and $7.8 \mathrm{~nm}$

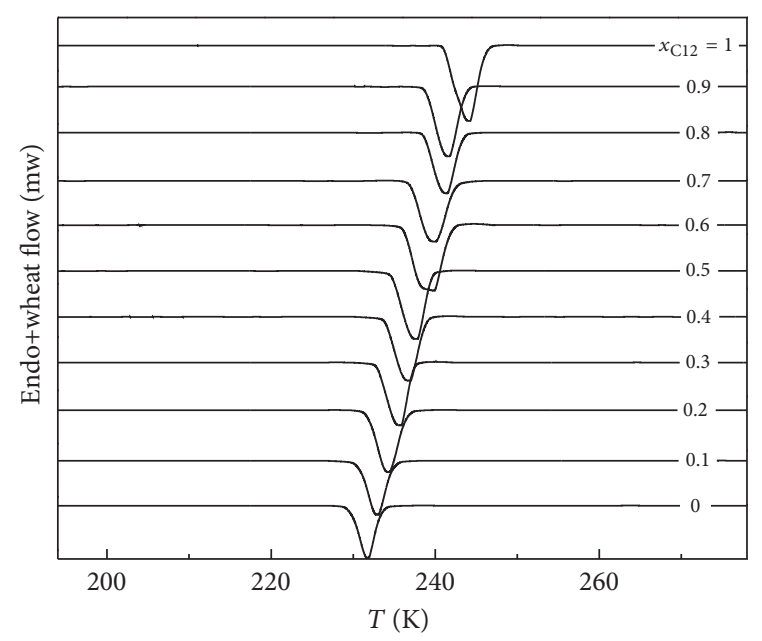

Figure 6: DSC curves of $\mathrm{C}_{11}-\mathrm{C}_{12}$ system confined in SBA-15 $(7.8 \mathrm{~nm})$ as a function of mole fractions of $\mathrm{C}_{12}\left(x_{\mathrm{C} 12}\right)$.

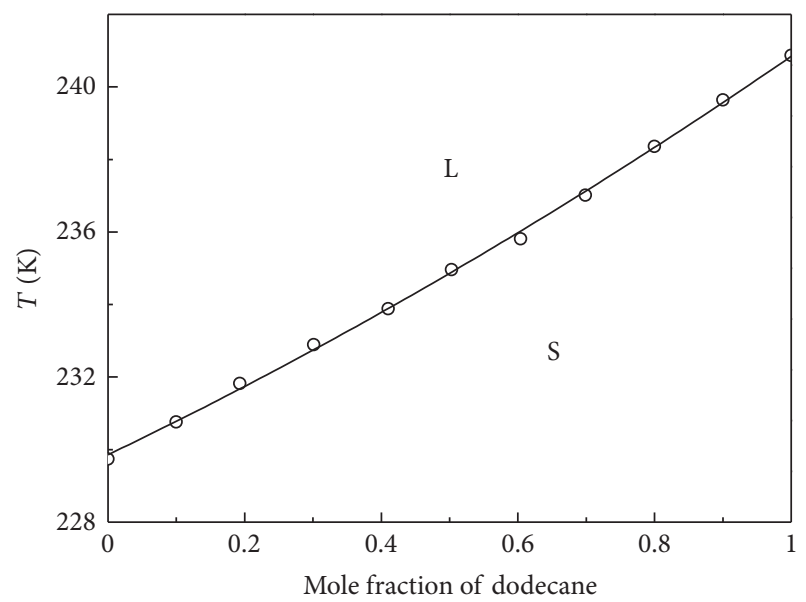

Figure 7: Experimental phase diagram of $\mathrm{C}_{11}-\mathrm{C}_{12}$ system confined in SBA-15 $(7.8 \mathrm{~nm})$.

would partially crystallize on cooling under the criterion of 10 or $20 \sigma$ for complete crystallization $[3,6,7]$.

Meanwhile, the alkane within pore of a size $17.2 \mathrm{~nm}$ could crystallize completely in consideration of a size of $20 \mathrm{~nm}$ for complete crystallization [6]. It is in this difference in the size of alkane liquids that might lead to distinct phase behavior among the above three systems.

According to the DSC curves of $\mathrm{C}_{11}-\mathrm{C}_{12} / \mathrm{SBA}-15$ $(17.2 \mathrm{~nm})$, the size of $17.2 \mathrm{~nm}$ may close to a threshold value that confined mixtures start to possess solid-solid transition property. In this case, the DSC curve shows weak but definite endothermic signal in the low-temperature region. A larger pore size should make the s-s transition more prominent. That would be the interest in next stage.

3.4. Melting Points of $C_{11}, C_{12}$ and the Mixtures as a Function of Pore Size. In Figure 10, the melting points $\left(T_{m, d}\right)$ are displayed for pure $\mathrm{C}_{11}, \mathrm{C}_{12}$ and the mixtures at $x_{\mathrm{C} 12}=0-1$ 


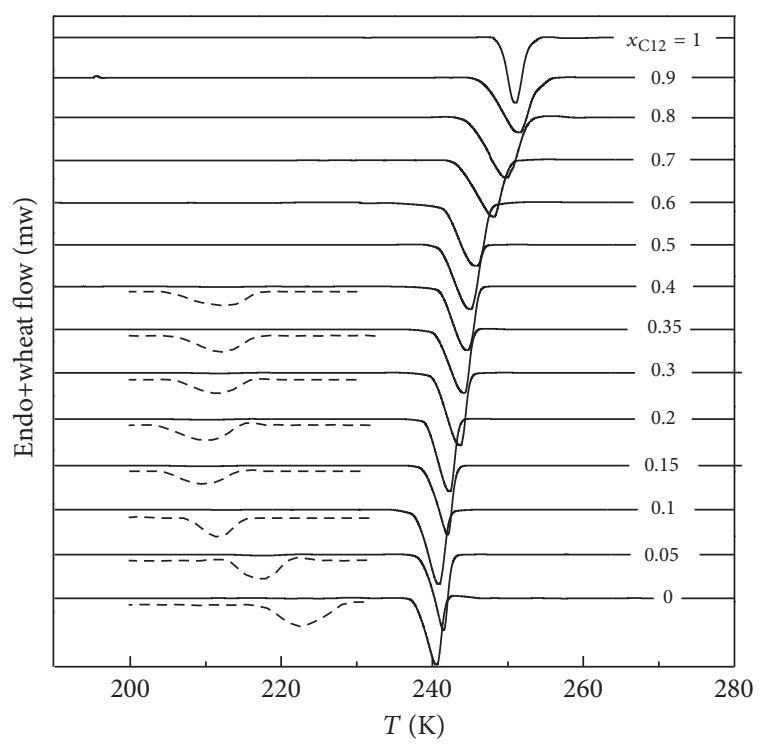

FIgURE 8: DSC curves of $\mathrm{C}_{11}-\mathrm{C}_{12}$ system confined in SBA-15 $(17.2 \mathrm{~nm})$ as a function of mole fractions of $x_{\mathrm{C} 12}$. From 200 to $230 \mathrm{~K}$, the solid-solid transitions are enlarged as the dash lines, next to the main DSC curves at $x_{\mathrm{C} 12}=0-0.4$ in aid of observation.

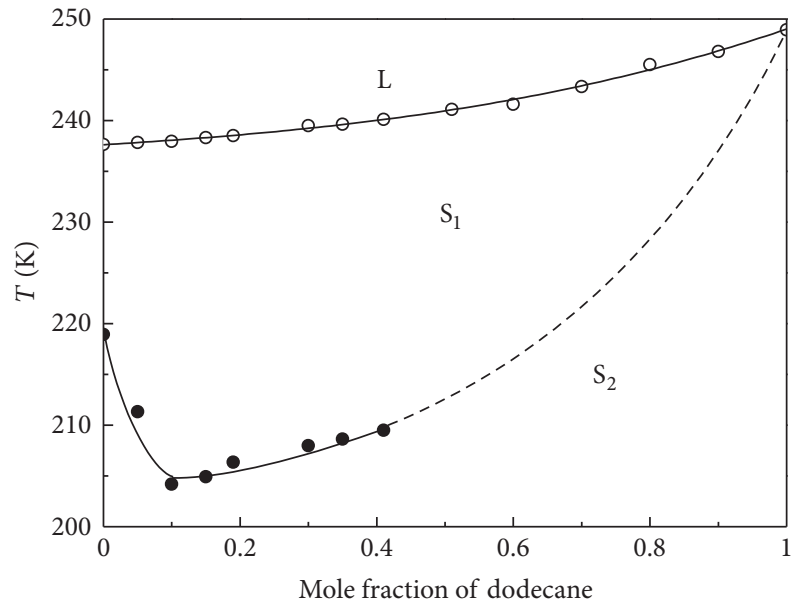

FIgURE 9: Experimental phase diagram of $\mathrm{C}_{11}-\mathrm{C}_{12}$ system confined in SBA-15 $(17.2 \mathrm{~nm})$. ○ experimental melting points at mole fractions $x_{\mathrm{C} 12} ; \bullet$ solid-solid transition temperatures.

confined in SBA-15 of pore diameter $d=(3.8,7.8$, and 17.2) $\mathrm{nm}$, respectively.

In the experiments, melting points of $\mathrm{C}_{11}, \mathrm{C}_{12}$ confined in SBA-15 (3.8, 7.8 and $17.2 \mathrm{~nm})$ are depressed compared with the bulk. This may be understood qualitatively on basis of previous works by Gubbins and coworkers (1) $[6,31,32]$ and a modified G-T equation described in [11] as follows:

$$
\begin{gathered}
T_{\mathrm{tr}}^{*} \approx f\left(H^{*}, \alpha\right), \\
\Delta T_{m}=T_{m, \text { bulk }}-T_{m, r}=\frac{2 V T_{m, \text { bulk }}\left(\gamma_{\mathrm{cw}}-\gamma_{\mathrm{lw}}\right)}{r \Delta H_{m}},
\end{gathered}
$$

where in (1) transition temperature (freezing/melting) $T_{\text {tr }}^{*}$ is

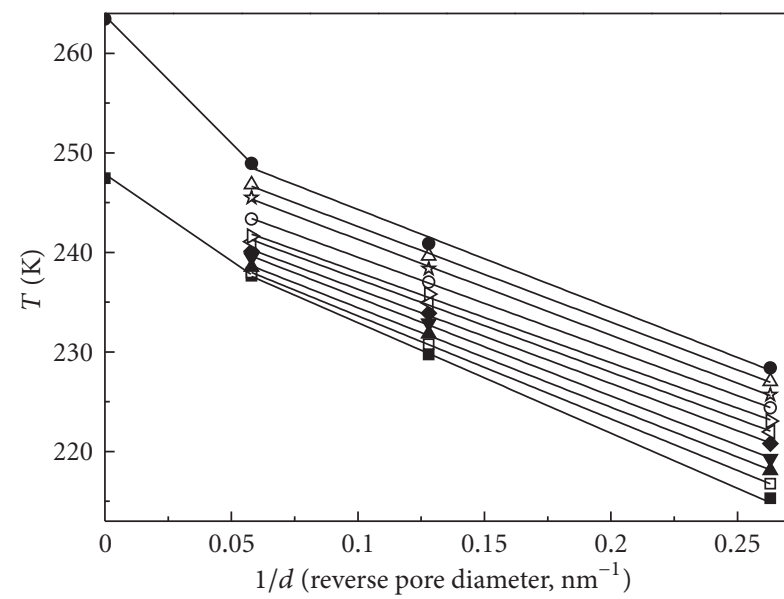

Figure 10: Melting points of pure $\left(\mathrm{C}_{11} ; \mathrm{C}_{12}\right), \mathrm{C}_{11}-\mathrm{C}_{12}$ system $\left(x_{\mathrm{C} 12}=\right.$ $0-1)$ confined in SBA-15 with pore diameter $d=(3.8,7.8$ and 17.2) $\mathrm{nm}$ - $\mathrm{C}_{11} ; \bullet \mathrm{C}_{12} ;$ mole fraction $x_{\mathrm{C} 12}: \square(0.1) ; \Delta(0.2) ; \nabla(0.3)$; $\checkmark(0.4) ; \triangleleft(0.5) ; \triangleright(0.6) ; \circ(0.7) ;$ 访 $(0.8) ; \Delta(0.9)$.

a function of the reduced pore size $H^{*}=H / \sigma$ ( $H$ is the pore width, and $\sigma$ is the diameter of pore liquid molecule) and the ratio of the wall/fluid (wf) to the fluid/fluid (ff) interactions, $\alpha \sim C \rho_{w} \varepsilon_{\mathrm{wf}} / \varepsilon_{\mathrm{ff}}$, where $\rho_{w}$ and $\varepsilon$ are the density of wall atoms and the potential well depth, respectively, and $\mathrm{C}$ is a constant depending on the wall geometry; in (2) $T_{m \text {,bulk }}$ is bulk melting point; $T_{m, r}$ is melting point of particle with radius $r ; V_{m}$ represents the crystal molar volume; $\gamma_{\mathrm{cw}}$ and $\gamma_{\mathrm{lw}}$ are crystalwall and liquid-wall interaction energy; $\Delta H_{m}$ the enthalpy of fusion of the crystal. As it is known, SBA-15 is comprised of silica tetrahedrons with nearly neutral or weakly polar pore wall because of surface hydroxyl groups. When nonpolar alkane molecules are adsorbed, an intermediate strength interaction produces between them. In this case, the value of $\alpha$ in (1) is less than 1 , that is, $\alpha<1$, which results in a depression of freezing/melting temperatures for $\mathrm{C}_{11}, \mathrm{C}_{12}$ confined in SBA-15, $[31,32]$. This also means that a relation exists, $\gamma_{\mathrm{cw}}>\gamma_{\mathrm{lw}}$, indicating in a depletion of transition temperatures either according to (2). From Figure 10, $T_{m, d}$ of $\mathrm{C}_{11}, \mathrm{C}_{12}$ shows a good linear relation with $1 / d$ over the size range from 3.8 to $17.2 \mathrm{~nm}, T_{m, d} \propto 1 / d$. Moreover, at each size $C_{11}$ melts in a lower temperature than $C_{12}$. This reflects the influence of $T_{m, \text { bulk }}, \Delta H_{m}$ and surface tension of the two alkanes besides the pore size.

It is clearly that the G-T equation cannot be used to predict phase behavior of $\mathrm{C}_{11}-\mathrm{C}_{12}$ binary system in confinement. However, in the scale of $3.8 \mathrm{~nm}$ to $17.2 \mathrm{~nm}$ in Figure 10, $T_{m, d}$ of the mixtures also shows a good linear relation with $1 / d$, $T_{m, d} \propto 1 / d$. The straight fitting lines for $T_{m, d}$ of the mixtures do not overlap and are almost parallel to each other. It seems that melting behavior of the mixtures can be compared to the pure alkane qualitatively under confinement.

In the size of 3.8 to $17.2 \mathrm{~nm}$, the $T_{m, d}$ of $\mathrm{C}_{11}, \mathrm{C}_{12}$ and the mixtures $\left(x_{\mathrm{C} 12}=0.1-0.9\right)$ are fitted as a function of $x_{\mathrm{C} 12}$ by the following equation:

$$
T_{m, d}\left(\mathrm{C}_{11}, \mathrm{C}_{12}\right)=\mathrm{C}_{1}-\frac{\mathrm{C}_{2}}{d},
$$


TABLE 1: Coefficients $\left(C_{1}, C_{2}\right)^{\mathrm{a}}$ in expression for melting points of $\mathrm{C}_{11}, \mathrm{C}_{12}$ and the mixtures $\left(x_{\mathrm{C} 12}\right)$ with pore diameter $(d)$ of $3.8 \sim 17.2$, and correlation coefficients $\left(R^{\mathrm{b}}\right)$.

\begin{tabular}{lcc}
\hline$x_{\mathrm{C} 12}$ & $C_{1}$ & $C_{2}$ \\
\hline $0\left(\mathrm{C}_{11}\right)$ & 243.83 & 108.70 \\
$1\left(\mathrm{C}_{12}\right)$ & 254.23 & 99.11 \\
0.100 & 243.98 & 103.52 \\
0.193 & 244.43 & 99.93 \\
0.301 & 245.35 & 98.89 \\
0.410 & 245.76 & 94.66 \\
0.503 & 246.69 & 93.67 \\
0.604 & 247.11 & 91.02 \\
0.699 & 248.77 & 92.63 \\
0.800 & 250.92 & 96.26 \\
0.900 & 252.19 & 95.98 \\
\hline
\end{tabular}

Melting point and pore diameter $d$ is fitted as $T_{m, d}=C_{1}-C_{2} / d$.

${ }^{\mathrm{b}}$ Correlation coefficients $R=0.9984 \sim 1.0000$.

where the pore diameter $d$ of SBA-15 is (3.8 to 17.2) nm; dimension of $C_{1}$ and $C_{2}$ is $\mathrm{Knm}^{-1}$. As for $\mathrm{C}_{12}, C_{1}=254.23$; $C_{2}=99.11 ; R \approx 0.9984 ;$ for $C_{11}, C_{1}=243.83 ; C_{2}=108.70$; $R \approx 0.9999$. The $C_{1}$ and $C_{2}$ values at each mole fractions are listed in Table 1.

3.5. Melting Points of $C_{11}-C_{12} / S B A-15(3.8,7.8$ and $17.2 \mathrm{~nm})$ as a Function of Composition. The experimental melting temperatures of $\mathrm{C}_{11}-\mathrm{C}_{12} / \mathrm{SBA}-15(3.8,7.8$ and $17.2 \mathrm{~nm})$ systems have been fitted as the following expressions:

(a) $\mathrm{C}_{11}-\mathrm{C}_{12} / \mathrm{SBA}-15(3.8 \mathrm{~nm})$ system: $T_{m, d}=12.8777 x+$ $215.43, R=0.9998$,

(b) $\mathrm{C}_{11}-\mathrm{C}_{12} / \mathrm{SBA}-15(7.8 \mathrm{~nm})$ system: $T_{m, d}=9.3898 x^{2}+$ $1.6199 x+229.83, R=0.9990$, and

(c) $\mathrm{C}_{11}-\mathrm{C}_{12} / \mathrm{SBA}-15(17.2 \mathrm{~nm})$ system: $T_{m, d}=9.3495 x^{2}+$ $1.7128 x+237.76, R=0.9952$,

where $T_{m, d}$ represents melting point of the mixture $(x) ; x$ is the mole fraction of $\mathrm{C}_{12}$. On basis of these linear or polynomial relations, melting points of a certain composition of $\mathrm{C}_{11}-\mathrm{C}_{12}$ system can be predicted at the three-pore size of $3.8,7.8$ and $17.2 \mathrm{~nm}$.

\section{Conclusion}

Physical size has a great influence on phase behavior of $\mathrm{C}_{11^{-}}$ $\mathrm{C}_{12}$ binary mixtures inside one-dimensional channels of SBA15. In the scale of 3.8 to $17.2 \mathrm{~nm}$, the solid-liquid phase diagram grows from a straight line $(3.8 \mathrm{~nm})$, a curve line $(7.8 \mathrm{~nm})$ to a loop line comprising of a curved solid-liquid and solid-solid boundary $(17.2 \mathrm{~nm})$. Melting temperatures of the mixtures with the three systems are depressed in a similar way with the pure $\mathrm{C}_{11}, \mathrm{C}_{12}$, which vary in consistent with mole fraction and pore diameter. The different behavior shows the dependence on the size of alkane.

\section{Acknowledgment}

The project was supported from Natural Science Foundation of Shandong Province, China (no. ZR2010BM035) and National Natural Science Foundation of China (no. 21273138).

\section{References}

[1] P. Buffat and J. P. Borel, "Size effect on the melting temperature of gold particles," Physical Review A, vol. 13, no. 6, pp. 2287-2298, 1976.

[2] C. J. Ellison and J. M. Torkelson, “The distribution of glasstransition temperatures in nanoscopically confined glass formers," Nature Materials, vol. 2, no. 10, pp. 695-700, 2003.

[3] P. Huber, V. Soprunyuk, and K. Knorr, "Structural transformations of even-numbered $n$-alkanes confined in mesopores," Physical Review E, vol. 74, Article ID 031610, 5 pages, 2006.

[4] A. Henschel, T. Hofmann, P. Huber, and K. Knorr, "Preferred orientations and stability of medium length $n$-alkanes solidified in mesoporous silicon," Physical Review E, vol. 75, Article ID 021607, 9 pages, 2007.

[5] D. Champion, C. Loupiac, D. Russo, D. Simatos, and J. M. Zanotti, "Dynamic and sub-ambient thermal transition relationships in water-sucrose solutions: differential scanning calorimetry and neutron scattering analysis," Journal of Thermal Analysis and Calorimetry, vol. 104, no. 1, pp. 365-374, 2011.

[6] C. Alba-Simionesco, B. Coasne, G. Dosseh et al., "Effects of confinement on freezing and melting," Journal of Physics Condensed Matter, vol. 18, no. 6, pp. R15-R68, 2006.

[7] S. Amanuel, H. Bauer, P. Bonventre, and D. Lasher, "Nonfreezing interfacial layers of cyclohexane in nanoporous silica," Journal of Physical Chemistry C, vol. 113, no. 44, pp. 18983-18986, 2009.

[8] M. Sliwinska-Bartkowiak, M. Jazdzewska, K. E. Gubbins, and L. Huang, "Melting behavior of bromobenzene within carbon nanotubes," Journal of Chemical and Engineering Data, vol. 55, no. 10, pp. 4183-4189, 2010.

[9] A. Toda, C. Tomita, T. Arita, and M. Hikosaka, "Periodically modulated driving force applied with TMDSC to the crystallization and melting kinetics of ice crystals confined in a porous silica gel," Journal of Thermal Analysis and Calorimetry, vol. 64, no. 2, pp. 775-782, 2001.

[10] M. Sha, G. Wu, Y. Liu, Z. Tang, and H. Fang, "Drastic phase transition in ionic liquid [Dmim] [CI] confined between graphite walls: new phase formatio," The Journal of Physical Chemistry, vol. 113, pp. 4618-4622, 2009.

[11] G. Dosseh, Y. Xia, and C. Alba-Simionesco, "Cyclohexane and benzene confined in MCM-41 and SBA-15: confinement effects on freezing and melting," Journal of Physical Chemistry B, vol. 107, no. 26, pp. 6445-6453, 2003.

[12] Y. I. Aristov, G. D. Marco, M. M. Tokarev, and V. N. Parmon, "Selective Water Sorbents for Multiple Applications, $\mathrm{CaCl}_{2}$ Solution Confined in Micro-and Mesoporous Silica Gels: Pore Size Effect on the "Solidification-Melting" Diagram," Elsevier Science BV, vol. 61, pp. 147-154, 1997.

[13] J. Czwartos, M. Sliwinska-Bartkowiak, B. Coasne, and K. E. Gubbins, "Melting of mixtures in silica nanopores," Pure and Applied Chemistry, vol. 81, no. 10, pp. 1953-1959, 2009.

[14] X. Z. Lan, H. R. Pei, and C. X. Cheng, "Phase behavior of binary system of bromobenzene-chlorobenzene confined in SBA-15 
and MCM-41," Chinese Chemical Letters, vol. 22, no. 12, pp. 1497-1500, 2011.

[15] X. Z. Lan, H. R. Pei, X. Yan, and W. B. Liu, "Phase behavior of dodecane-tetradecane binary system confined in SBA-15," Journal of Thermal Analysis and Calorimetry. In press.

[16] H. R, X. Yan, W. B. Liu, and X. Z. Lan, "Phase behavior of tetradecane-hexadecane mixtures confined in SBA-15," Journal of Thermal Analysis and Calorimetry. In press.

[17] D. Fu, Y. Liu, G. Liu, Y. Su, and D. Wang, "Confined crystallization of binary $n$-alkane mixtures: stabilization of a new rotator phase by enhanced surface freezing and weakened intermolecular interactions," Physical Chemistry Chemical Physics, vol. 13, pp. 15031-15036, 2011.

[18] P. Espeau, L. Robles, M. A. Cuevas-Diarte, D. Mondieig, and Y. Haget, "Thermal cycling of molecular alloys and eutectics containing alkanes for energy storage," Materials Research Bulletin, vol. 31, no. 10, pp. 1219-1232, 1996.

[19] D. B. Andrew and E. D. John, Acta Crystallographica Section E, vol. 58, p. 196, 2002.

[20] D. K. Pan, C. D. Zhao, and Z. X. Zheng, Structure Chemistry, Higher Education press, Beijing, China, 1st edition, 1987.

[21] D. Mondieig, P. Espeau, L. Robles, Y. Haget, H. A. J. Oonk, and M. A. Cuevas-Diarte, "Mixed crystals of n-alkane pairs: a global view of the thermodynamic melting properties," Journal of the Chemical Society, vol. 93, no. 18, pp. 3343-3346, 1997.

[22] L. Ventolà, T. Calvet, M. Á. Cuevas-Diarte, V. Métivaud, D. Mondieig, and H. Oonk, "From concept to application. A new phase change material for thermal protection at $-11^{\circ} \mathrm{C}$," Materials Research Innovations, vol. 6, no. 5-6, pp. 284-290, 2002.

[23] Y. H. Yan, F. F. Li, C. Yang, W. Y. Zhang, and J. H. Wu, "Grafting Morphologies of TEPA on SBA-15(P) and Its Effect on $\mathrm{CO}_{2}$ Adsorption Performance," Acta Physica Sinica, vol. 28, pp. 195-200, 2012.

[24] L. Cao, T. Man, and M. Kruk, "Synthesis of ultra-largepore SBA-15 silica with two-dimensional hexagonal structure using triisopropylbenzene as micelle expander," Chemistry of Materials, vol. 21, no. 6, pp. 1144-1153, 2009.

[25] M. Imperor-Clerc, P. Davidson, and A. Davidson, "Existence of a microporous corona around the mesopores of silicabased SBA-15 materials templated by triblock copolymers," Journal of the American Chemical Society, vol. 122, no. 48, pp. 11925-11933, 2000.

[26] S. Y. Liu, X. J. Meng, and F. S. Xiao, "Hydrothermal synthesis of ordered mesoporous materials with high stability at high temperatures," Acta Physico-Chimica Sinica, vol. 26, no. 7, pp. 1852-1859, 2010.

[27] M. Kruk, M. Jaroniec, and A. Sayari, "Application of large pore MCM-41 molecular sieves to improve pore size analysis using nitrogen adsorption measurements," Langmuir, vol. 13, no. 23, pp. 6267-6273, 1997.

[28] T. Calvet, E. Tauler, M. A. Cuevas-Diarte et al., "Application of the "shape-factors method" to purity analysis of compounds by thermal methods," Thermochimica Acta, vol. 204, no. 2, pp. 271-280, 1992.

[29] F. D. Joeri, B. Abdelkarim, and G. V. Baron, "Adsorption of alkanes and other organic molecules in liquid phase and in the dense vapor phase: influence of polarity, zeolite topology, and external fluid density and pressure," Industrial \& Engineering Chemistry Research, vol. 37, no. 9, pp. 3691-3698, 1998.
[30] J. F. Denayer, J. F. M, D. M. Kurt, A. M. Johan, and G. V. Baron, "Molecular competition effects in liquid-phase adsorption of long-chain $n$-alkane mixtures in ZSM-5 zeolite pores," Angewandte Chemie, vol. 115, no. 24, pp. 2880-2883, 2003.

[31] B. Coasne, J. Czwartos, M. Sliwinska-Bartkowiak, and K. E. Gubbins, "Effect of pressure on the freezing of pure fluids and mixtures confined in nanopores," Journal of Physical Chemistry $B$, vol. 113, no. 42, pp. 13874-13881, 2009.

[32] B. Coasne, J. Czwartos, M. Sliwinska-Bartkowiak, and K. E. Gubbins, "Freezing of mixtures confined in silica nanopores: experiment and molecular simulation," Journal of Chemical Physics, vol. 133, no. 8, Article ID 084701, 2010. 

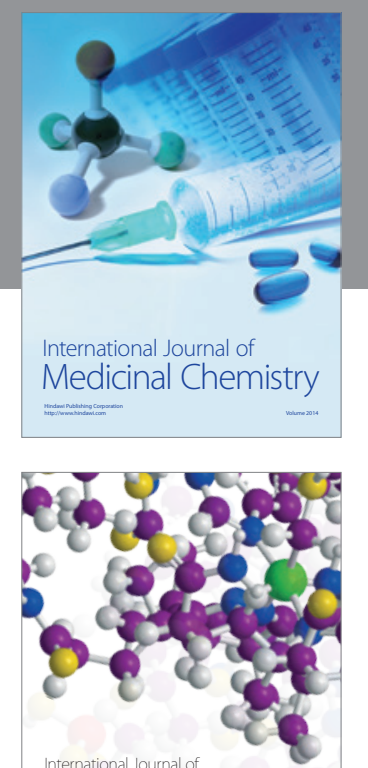

\section{Carbohydrate} Chemistry

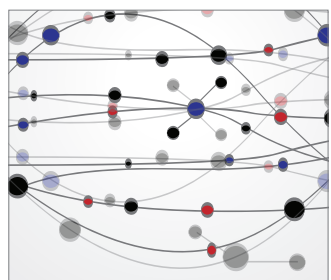

The Scientific World Journal
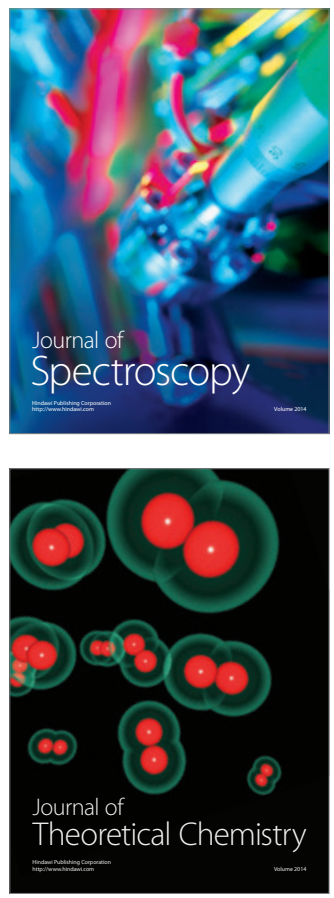
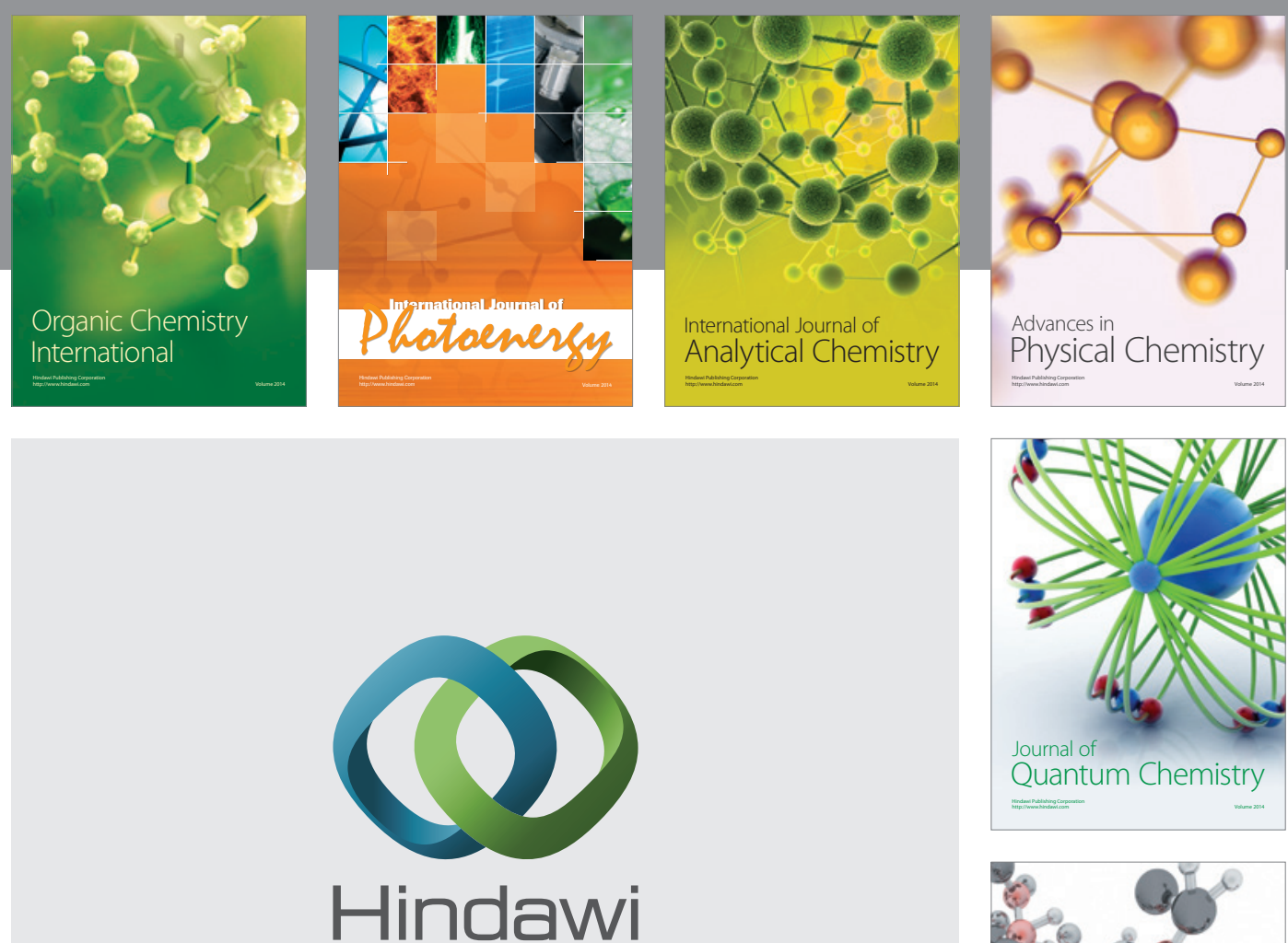

Submit your manuscripts at

http://www.hindawi.com

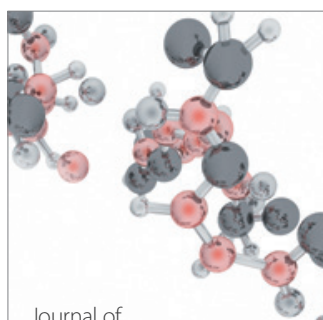

Analytical Methods

in Chemistry

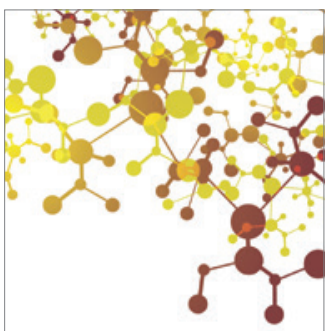

Journal of

Applied Chemistry

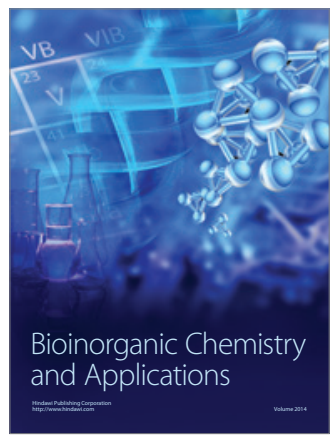

Inorganic Chemistry
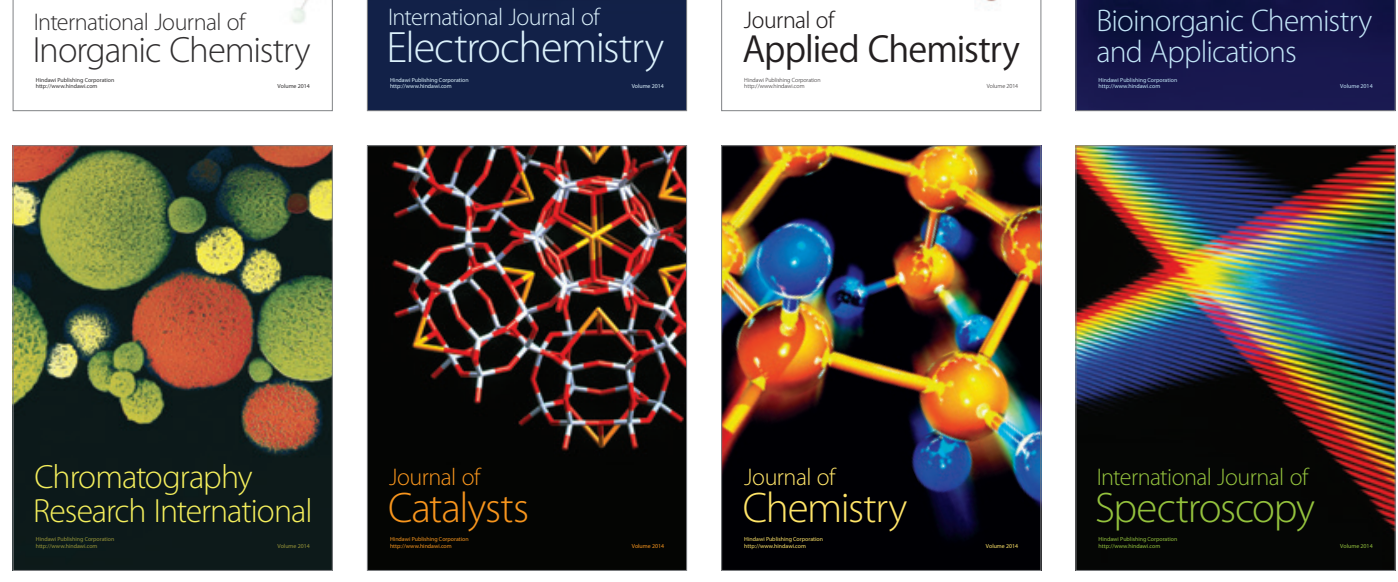